\title{
Impact of Rheumatoid Arthritis on Body Image Disturbance
}

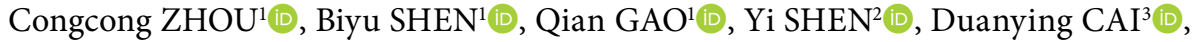 \\ Zhifeng $\mathrm{GU}^{4} \mathbb{D}$, Xujuan $\mathrm{XU}^{1}{ }^{1}$ \\ ${ }^{1}$ Department of Nursing, The Affiliated Hospital of Nantong University, Nantong, China \\ ${ }^{2}$ Nantong University, School of Epidemiology and Medical Statistics, Nantong, China \\ ${ }^{3}$ Nantong University, School of Nursing, Nantong, China \\ ${ }^{4}$ Department of Rheumatology, The Affiliated Hospital of Nantong University, Nantong, China
}

\begin{abstract}
Objectives: This study aims to explore body image disturbance (BID) in Chinese patients with rheumatoid arthritis (RA).

Patients and methods: A total of 211 RA patients (30 males, 181 females; mean age $50.4 \pm 12.4$ years; range, 20 to 81 years) and 203 age-matched healthy subjects ( 26 males, 177 females; mean age $49.6 \pm 5.6$ years; range, 34 to 64 years) were included in this study. Patients were assessed by Body Image Disturbance Questionnaire (BIDQ), Visual Analog Scale, Health Assessment Questionnaire-Disability Index, disease activity score in 28 joints, Short Form 36 health survey, Hospital Anxiety and Depression Scale, Rosenberg Self-Esteem Scale and erythrocyte sedimentation rate.

Results: The mean disease duration of RA patients was $7.2 \pm 8.2$ years. BID occurrence in RA patients was reported in $24.2 \%$ of the cases, which was significantly higher than the healthy subjects with $3 \%$. Stepwise logistic regression analysis demonstrated that social function $(p<0.05)$ and anxiety $(p<0.01)$ explained higher BIDQ score.
\end{abstract}

Conclusion: Body image disturbance was common in Chinese RA patients. Rheumatologists and nurses should attach great importance to BID in RA patients, particularly in those with poor social function and anxiety, by examining their physical limitations and psychological disability.

Keywords: Body image disturbance; Chinese patients; rheumatoid arthritis.

Rheumatoid arthritis (RA) is an autoimmune disease affecting $0.32-0.38 \%$ of the world's population with prominent manifestations in the joints characterized by pain, swelling, and stiffness. ${ }^{1-3}$ With the progress of disease in RA, some patients may have irreversible damage like decreased mobility and functional restrictions which may cause psychological problems such as body image disturbance (BID) , $^{4}$ BID is a kind of severe emotional distress experienced by people with a negative perception of the body, which may impair one's physical and psychological health, ${ }^{6-8}$ A healthy body image is believed to be good for one's health-related quality of life (HRQOL). ${ }^{9}$ Previous studies indicated that many chronic diseases, particularly those characterized by deformity and disability, are generally associated with BID. Interestingly, few rheumatologists focus on how RA affects the patient's perception of his/her own body and how these body changes may affect the patient's psychological and physical health. Most rheumatologists tend to focus on reducing inflammation and disability of limiting symptoms. ${ }^{10}$ All of these factors highlight the fact that BID needs to be considered to improve the overall HRQOL in RA patients.

Received: November 15, 2017 Accepted: May 04, 2018 Published online: August 16, 2018

Correspondence: Xujuan Xu, MD. Department of Rheumatology, The First Affiliated Hospital of Nantong University, 226001 Nantong, China. Tel: 8615851218899 e-mail: ntuxxj_1124@126.com

\section{Citation:}

Zhou C, Shen B, Gao Q, Shen Y, Cai D, Gu Z, et al. Impact of rheumatoid arthritis on body image disturbance. Arch Rheumatol 2019;34(1):79-87. 
There is limited quantitative research focused on body image of RA patients, most current studies being qualitative research. ${ }^{11-14}$ Early research on BID of RA patients stated that RA patients often admitted that they were less charming than healthy control groups. ${ }^{15,16}$ It has been recognized that a number of factors, such as demographic variables (e.g.: sex and socioeconomic status), ${ }^{14,17}$ physical factors (e.g.: pain and functional disability), ${ }^{12,17}$ and psychological issues (e.g.: anxiety and depression) $)^{4,10}$ were significantly related to BID. However, few studies focused on disease duration, disease activity, morning stiffness time, laboratory indicators and BID in RA patients. Furthermore; point prevalence, contributors and predictors of BID in China are also unclear. For this reason, in this study, we aimed to explore BID in Chinese patients with RA.

\section{PATIENTS AND METHODS}

In this cross-sectional study, a total of 211 RA patients (30 males, 181 females; mean age $50.4 \pm 12.4$ years; range, 20 to 81 years) and 203 age-matched healthy subjects (26 males, 177 females; mean age 49.6 \pm 5.6 years; range, 34 to 64 years) were screened and enrolled from the Affiliated hospital of Nantong University between November 2016 and April 2017. Healthy subjects were screened in the physical examination center. Inclusion criteria were: (i) age ( $\geq 18$ years); (ii) definite diagnosis of RA according to 2010/2012 American College of Rheumatology criteria; ${ }^{18}$ and (iii) having no complications (e.g., serious respiratory, cardiac, gastrointestinal, endocrine, neurological diseases or infections), which may influence the outcome of the study. Healthy subjects were excluded if they were not in good health or reported major systematic diseases or psychological disorders. The study protocol was approved by the Ethics Committee of the Affiliated Hospital of Nantong University. A written informed consent was obtained from each patient. The study was conducted in accordance with the principles of the Declaration of Helsinki.

Demographic characteristics included age, sex, body mass index, education, marital status, average annual income, occupation, smoking and alcohol use. Morning stiffness time, disease duration and erythrocyte sedimentation rate (ESR, mm/hour) were recorded based on medical records or patients' direct responses.

Patients' body image perception was measured by the Body Image Disturbance Questionnaire (BIDQ) with seven items. The seven items include perceived concern, preoccupation, role avoidance and emotional distress of their own appearance, and appearance impairment in social, education and work function. Each item is scaled between 1 ('no effect') and 5 ('totally affected') and clinicians often judge BID by a mean score of BIDQ >3.0. ${ }^{19}$ The simplified Chinese version of BIDQ had an acceptable internal consistency and a Cronbach's alpha value of $0.877 . .^{20}$

A $10 \mathrm{~cm}$ Visual Analog Scale was used to measure perceived pain. All patients rated their feelings of perceived pain within one week from 0 (not at all) to 10 (extremely affected). Higher score indicated more severe pain. ${ }^{21}$

The Health Assessment QuestionnaireDisability Index (HAQ-DI) is a reliable and valid instrument to measure patients' perceived difficulty in completing daily activities. This questionnaire contains 20 items scaled between 0 (no difficulty) and 3 (completely disabled), with higher scores representing severe dysfunction. ${ }^{22}$ The Cronbach's alpha value in the Chinese version of HAQ-DI is 0.86 . The test-retest reliability was surveyed to be between $0.54-0.80 .{ }^{23}$

Disease activity score in 28 joints (DAS28) was used to measure patient's disease activity. This questionnaire contains four aspects which was calculated by a software with 28 swollen joint counts, 28 tender joint counts, ESR and patient's perception of disease activity from $0 \mathrm{~mm}$ (not active at all) to $100 \mathrm{~mm}$ (extremely active). ${ }^{24}$

Participant's health status was measured by the Short Form 36 (SF-36) health survey in the past one month. This questionnaire includes two main domains of mental component summary (MCS) and physical component summary (PCS). MCS consists of four domains including energy/vitality (VT), social function (SF), role limitations, emotional problems and mental health while PCS contains physical function, role limitations, body pain and general health perception. All domains are scored between 0 and 100, a higher score indicating better health status. ${ }^{25}$ The Chinese version of SF-36 


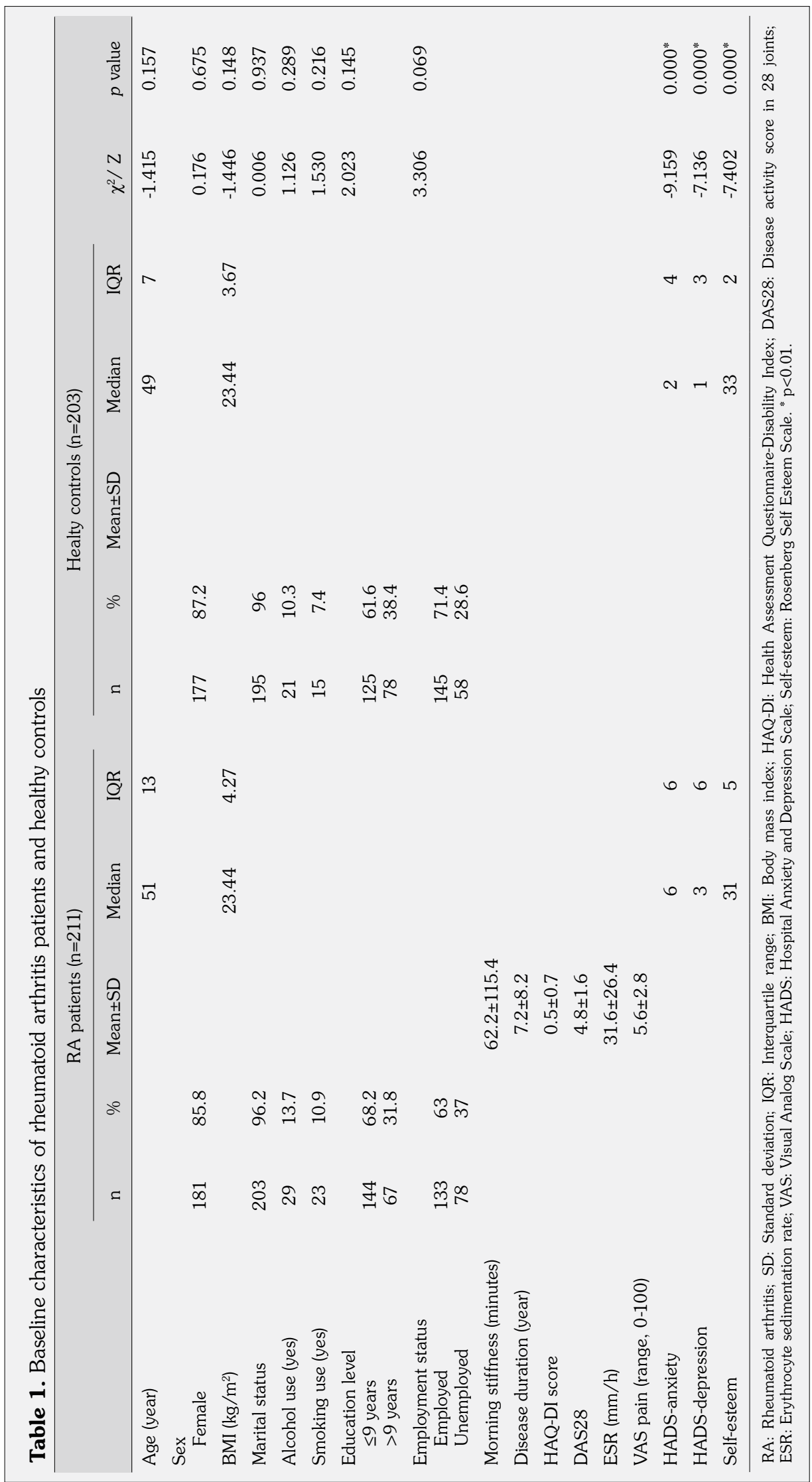


with Cronbach's alpha ranged from 0.72 to 0.88 except 0.66 for VT and 0.39 for social function. The test-retest reliability coefficients after two weeks were between 0.66 and $0.94 .{ }^{26}$

The Hospital Anxiety and Depression Scale (HADS) contains two subscales used to measure anxiety and depression in the past one month. Each subscale has seven items, and every item is scored from $0-3$; so, the summation of each subscale is scored between 0 and $21 .{ }^{27}$ The Chinese version of HADS has been widely used in Mainland China. The Cronbach's alpha value of this questionnaire is 0.85 and the intraclass correlation coefficient is $0.90 .^{28}$

The Rosenberg Self-Esteem Scale was used to assess participants' attitude of self-esteem. The scale has ten items of a total score of 30 points, with higher scores representing higher level of self-esteem. ${ }^{29}$

\section{Statistical analysis}

Continuous and normally distributed variables were presented as mean \pm standard deviation (SD) and group differences were measured by independent sample t-test. Not normally distributed data were described by median and interquartile range and group differences were assessed by Mann-Whitney U test. Descriptive statistics also involved frequencies (\%) for categorical variables and the group differences were measured by chi-square test. Variables shown to be significant in the independent sample were included into the stepwise logistic regression model with the BID which was measured by the BIDQ as the dependent variable. A p value of $<0.05$ (two-sided) was considered statistically significant. Data were analyzed using IBM SPSS version 20.0 software program (IBM Corp., Armonk, NY, USA).

Table 2. Comparison of body image disturbance and health related quality of life for rheumatoid arthritis patients and healthy controls

\begin{tabular}{|c|c|c|c|c|c|c|c|c|c|c|}
\hline & \multicolumn{4}{|c|}{ RA patients $(\mathrm{n}=211)$} & \multicolumn{4}{|c|}{ Healthy controls $(n=203)$} & \multirow[b]{2}{*}{$\chi^{2} / Z$} & \multirow[b]{2}{*}{$\mathrm{p}$ value } \\
\hline & $\mathrm{n}$ & $\%$ & Median & IQR & $\mathrm{n}$ & $\%$ & Median & IQR & & \\
\hline \multicolumn{11}{|l|}{ BIDQ assessments } \\
\hline Appearance-related concerns & & & 4 & 4 & & & 4 & 0 & -0.293 & 0.770 \\
\hline Appearance preoccupation & & & 2 & 4 & & & 2 & 2 & -1.467 & 0.142 \\
\hline Distress & & & 2 & 4 & & & 0 & 2 & -6.718 & $0.000^{*}$ \\
\hline Social function impairment & & & 0 & 2 & & & 0 & 0 & -6.522 & $0.000^{*}$ \\
\hline Social life impairment & & & 0 & 2 & & & 0 & 0 & -7.231 & $0.000^{*}$ \\
\hline Role function impairment & & & 0 & 2 & & & 0 & 0 & -8.405 & $0.000^{*}$ \\
\hline Role avoidance & & & 0 & 2 & & & 0 & 0 & -4.737 & $0.000^{*}$ \\
\hline Mean BID score & & & 1.71 & 1.86 & & & 1.14 & 0.57 & -5.873 & $0.000^{*}$ \\
\hline BID & 51 & 24.2 & & & 6 & 3 & & & 39.221 & $0.000^{*}$ \\
\hline \multicolumn{11}{|l|}{ QOL assessments } \\
\hline $\mathrm{PF}$ & 70 & 40 & & & & & 95 & 10 & -12.666 & $0.000^{*}$ \\
\hline $\mathrm{RP}$ & 0 & 50 & & & & & 100 & 25 & -13.167 & $0.000^{*}$ \\
\hline $\mathrm{BP}$ & 41 & 50 & & & & & 100 & 16 & -16.190 & $0.000^{*}$ \\
\hline $\mathrm{GH}$ & 40 & 30 & & & & & 70 & 17 & -12.264 & $0.000^{*}$ \\
\hline VT & 50 & 25 & & & & & 70 & 20 & -9.900 & $0.000^{*}$ \\
\hline $\mathrm{SF}$ & 62.5 & 37.5 & & & & & 100 & 13 & -12.745 & $0.000^{*}$ \\
\hline $\mathrm{RE}$ & 0 & 100 & & & & & 100 & 0 & -10.740 & $0.000^{*}$ \\
\hline $\mathrm{MH}$ & 60 & 28 & & & & & 76 & 12 & -8.398 & $0.000^{*}$ \\
\hline PCS & 40.5 & 25 & & & & & 91.25 & 14.25 & -15.175 & $0.000^{*}$ \\
\hline MCS & 50.13 & 40.3 & & & & & 85.5 & 12.75 & -12.139 & $0.000^{*}$ \\
\hline
\end{tabular}

RA: Rheumatoid arthritis; IQR: Interquartile range; BIDQ: Body image disturbance (questionnaire); HRQOL: Health-related quality of life; PF: Physical function; RP: Role limitations due to physical problems; BP: Body pain; GH: General health perception; VT: Energy/vitality; SF: Social function; RE: Role limitations due to emotional problems; MH: Mental health; PCS: Physical component summary; MCS: Mental component summary. ${ }^{*} \mathrm{p}<0.01$ 


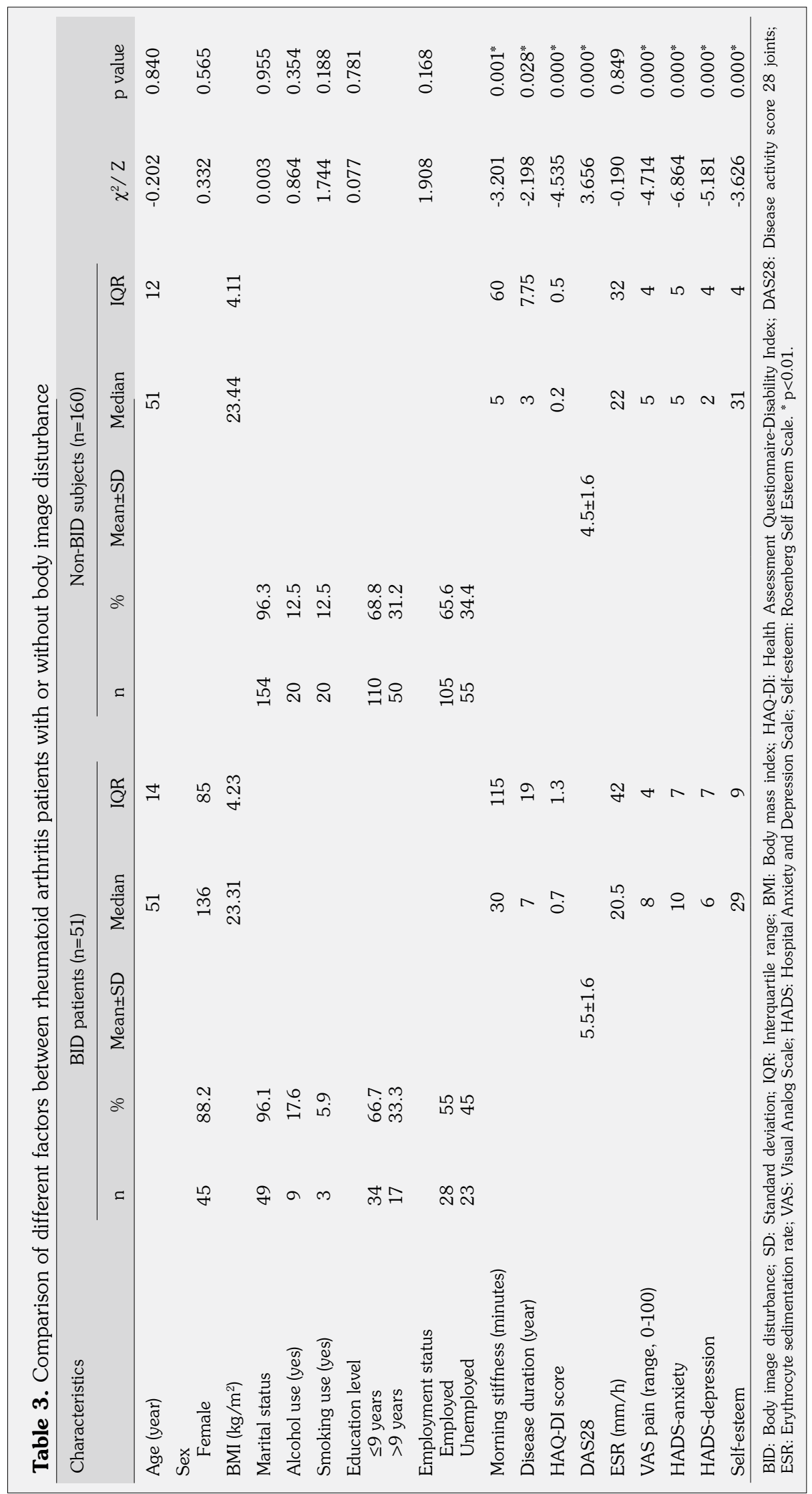


Table 4. Comparison of health related quality of life between rheumatoid arthritis patients with or without body image disturbance

\begin{tabular}{|c|c|c|c|c|c|c|}
\hline \multirow[t]{2}{*}{ Characteristics } & \multicolumn{2}{|c|}{ BID patients $(n=51)$} & \multicolumn{2}{|c|}{ Non-BID subjects $(n=160)$} & \multirow[t]{2}{*}{ Z } & \multirow[t]{2}{*}{$p$ value } \\
\hline & Median & IQR & Median & IQR & & \\
\hline $\mathrm{PF}$ & 50 & 45 & 75 & 33.75 & -5.669 & $0.000^{*}$ \\
\hline $\mathrm{RP}$ & 0 & 0 & 0 & 75 & -4.714 & $0.000^{*}$ \\
\hline BP & 22 & 19 & 51 & 31 & -5.587 & $0.000^{*}$ \\
\hline $\mathrm{GH}$ & 30 & 15 & 45 & 30 & -4.417 & $0.000^{*}$ \\
\hline VT & 40 & 25 & 55 & 30 & -4.473 & $0.000^{*}$ \\
\hline SF & 37.5 & 37.5 & 75 & 37.5 & -6.057 & $0.000^{*}$ \\
\hline $\mathrm{RE}$ & 0 & 0 & 50 & 100 & -4.673 & $0.000^{*}$ \\
\hline MH & 48 & 36 & 64 & 24 & -4.559 & $0.000^{*}$ \\
\hline SF-36 PCS & 29.25 & 16.5 & 48 & 30.63 & -6.893 & $0.000^{*}$ \\
\hline SF-36 MCS & 31.88 & 23.5 & 61.34 & 35.9 & -6.156 & $0.000^{*}$ \\
\hline
\end{tabular}

\section{RESULTS}

Table 1 presents the baseline characteristics of all participants. In RA patients, $85.8 \%$ were females and the mean age (SD) was $50.4 \pm 12.4$ years. There was no significant difference in social demographics between RA and control groups ( $p>0.05$ ). Significant difference existed between two groups in anxiety, depression and self-esteem $(p<0.01)$.

As shown in Table 2, no significant differences were observed in patients' perceived concern and preoccupation of appearance dimensions from BIDQ between RA and control groups ( $>0.05$ ). RA patients exhibited more impairment for other items in BIDQ $(p<0.01)$. A comparison of quality of life between two groups revealed that all domains of HRQOL from SF-36 in RA group were significantly reduced compared to the control group $(p<0.01)$.

Table 3 reveals a number of differences between RA patients with or without BID. RA patients with BID had longer morning stiffness time, longer disease duration, more perceived pain, higher level of depression, anxiety and disease activity $(p<0.01)$. RA patients with BID tended to have lower functional capacity and self-esteem compared to those without BID $(p<0.01)$.

Scores from SF-36 questionnaire of RA patients with BID were lower than those without

Table 5. Logistic regression analysis of variables in relation to body image disturbance in rheumatoid arthritis patients

\begin{tabular}{|c|c|c|c|c|c|c|}
\hline \multirow[b]{2}{*}{ Variables } & \multirow[b]{2}{*}{$\mathrm{B}$} & \multirow[b]{2}{*}{ SE } & \multirow[b]{2}{*}{$\mathrm{p}$} & \multirow[b]{2}{*}{$\operatorname{Exp}(B)$} & \multicolumn{2}{|c|}{$95 \% \mathrm{CI}$} \\
\hline & & & & & Lower & Higher \\
\hline $\mathrm{BP}$ & 0.023 & 0.013 & 0.079 & 1.024 & 0.997 & 1.051 \\
\hline SF & 0.025 & 0.010 & $0.015^{*}$ & 1.026 & 1.005 & 1.047 \\
\hline HADS-A & -0.312 & 0.089 & $0.000^{* * *}$ & 0.732 & 0.615 & 0.871 \\
\hline HADS-D & 0.157 & 0.089 & 0.079 & 1.170 & 0.982 & 1.394 \\
\hline Constant & 0.425 & 0.843 & 0.254 & 1.530 & & \\
\hline \multicolumn{7}{|c|}{ Nagelkerke $R^{2}=0.441$} \\
\hline
\end{tabular}


BID in terms of physical function, role limitations, body pain, general health perception, energy, social function, role limitations due to emotional problems, mental health, PCS and MCS (Table 4) $(p<0.01)$.

We used stepwise multiple logistic regression analysis to investigate predictors of BID (Table 5). Only the variables shown to be significant $(p<0.05)$ were included in the model. We found that anxiety and social function domains of the SF-36 were predictors of BID.

\section{DISCUSSION}

Our study has explored BID comprehensively on the Chinese mainland patients with RA. BID is common with a prevalence of $24.2 \%$, while poor social function and anxiety were predictive factors of BID in RA patients.

Our results suggest that there is an obvious difference in BID occurrence between RA patients and control subjects. This is different from Cornwell and Schmitt's findings, ${ }^{16}$ where they used a number of measures to compare RA patients and control groups and stated that the two groups did not show any significant difference in body image. However, their groups were heterogeneous in terms of socio-demographic characteristics. Other studies reported the same conclusion as this study when it comes to BID of RA patients and controls. ${ }^{30}$ Similarly, Jorge et al. ${ }^{31}$ discovered a worse BID score in RA patients than in control groups. All of these findings highlight the fact that RA patients have poor body image perception compared to healthy people. Therefore, these findings are worth considering because BID might be overlooked by rheumatologists and nurses during treatment.

Previous studies have reported that RA patient's age, sex and education were closely associated with BID. ${ }^{31,32}$ In this study, we did not find any association between BID and these variables. The reason for these two different results may be caused by the different participants in China and Western countries and the existence of cultural diversity. As BID is a type of severe emotional distress which is related to the body, it is not surprising for people to judge their appearance based on the interactions they have in society. Furthermore, the beauty standards of the society may influence their thoughts profoundly. Therefore, it is common for patients to have psychological disorders such as low self-esteem if they do not achieve the standards they expect. Similar to the previous studies, our study serves as a proof for how low self-esteem is associated with BID. When it comes to other psychological dimensions such as anxiety and depression, which were broadly investigated in BID patients, we also detected similar conclusions as in most studies indicating that BID had a positive correlation with anxiety and depression. ${ }^{4,10}$ On the other hand, Monaghan et al. ${ }^{4}$ demonstrated no obvious difference between BID and anxiety in RA patients, which may be due to some cultural characters that might affect psychological dimensions in Chinese patients. Based on this, rheumatologists and nurses should attach more importance to psychological variables in RA patients.

Since joints are increasingly affected with the progress of RA, patients suffering from severe physical deformities and disabilities may have higher levels of BID. Hence a correlation was found between disease duration, morning stiffness time, DAS28 and BID. Previous studies indicated that poor physical capacity may contribute to a negative body image perception. ${ }^{31}$ In our study, functional capacity (social function domain of SF-36) was positively related to $\mathrm{BID}$ as well. One major symptom of RA is pain; accordingly, we found that body pain was closely associated with BID. Similarly, it has been reported in previous studies that pain is the most important indicator of BID. ${ }^{32}$

We used stepwise regression analysis to identify which variables were significantly associated with $\mathrm{BID}$. The results of our study indicated that anxiety and poor social function were independent risk factors of BID. However, as shown in Table 5, the reason why depression and pain symptoms were not the predictive factors of BID may be explained by the disease status of these patients being more severe which prevented such patients with severe pain or depression from having the time or vitality to consider BID.

Despite its important discoveries, our study has some limitations. First, our sample was from a single clinic of rheumatology based on selfreported questionnaires only, which may have easily caused bias in our outcomes. Second, this 
is a cross-sectional study, making it challenging to infer causal relationships. Third, we did not include the effects of drugs on BID of RA patients. Therefore, prospective studies with larger sample sizes are required regarding the effects of drugs on RA patients' BID.

In conclusion, RA patients had worse BID than healthy individuals. It is important for rheumatologists and nurses to attach attention to BID in RA patients, particularly in those with symptoms of anxiety or poor functional capacity.

\section{Declaration of conflicting interests}

The authors declared no conflicts of interest with respect to the authorship and/or publication of this article.

\section{Funding}

This work was supported by the Natural Science Foundation of China (Grant no. 81671616), the Humanistic Nursing Care Foundation of China (Grant no, RW2016AM14), Jiangsu Provincial Commission of Health and Family planning Foundation (Grant no, N2017008) and the Science Foundation of Nantong City (Grant no, ms12017009-5).

\section{REFERENCES}

1. Araki Y, Mimura T. The Mechanisms Underlying Chronic Inflammation in Rheumatoid Arthritis from the Perspective of the Epigenetic Landscape. J Immunol Res 2016;2016:6290682.

2. Kojima $\mathrm{M}$, Kojima $T$, Ishiguro $\mathrm{N}$, Oguchi $\mathrm{T}$, Oba $\mathrm{M}$, Tsuchiya $\mathrm{H}$, et al. Psychosocial factors, disease status, and quality of life in patients with rheumatoid arthritis. J Psychosom Res 2009;67:425-31.

3. Zhang L, Xia Y, Zhang Q, Fu T, Yin R, Guo G, et al. The correlations of socioeconomic status, disease activity, quality of life, and depression/anxiety in Chinese patients with rheumatoid arthritis. Psychol Health Med 2017:22:28-36.

4. Monaghan SM, Sharpe L, Denton F, Levy J, Schrieber L, Sensky T. Relationship between appearance and psychological distress in rheumatic diseases. Arthritis Rheum 2007;57:303-9.

5. Boers M. Understanding the window of opportunity concept in early rheumatoid arthritis. Arthritis Rheum 2003;48:1771-4.

6. Partridge KA, Robertson N. Body-image disturbance in adult dialysis patients. Disabil Rehabil 2011;33:504-10.

7. Cash TF, Phillips KA, Santos MT, Hrabosky JI. Measuring "negative body image": validation of the Body Image Disturbance Questionnaire in a nonclinical population. Body image 2004;1:363-72.

8. Dyer A, Borgmann E, Feldmann RE Jr, Kleindienst N, Priebe $\mathrm{K}$, Bohus $\mathrm{M}$, et al. Body image disturbance in patients with borderline personality disorder: impact of eating disorders and perceived childhood sexual abuse. Body Image 2013;10:220-5.

9. Bowe WP, Doyle AK, Crerand CE, Margolis DJ, Shalita AR. Body image disturbance in patients with acne vulgaris. J Clin Aesthet Dermatol 2011;4:35-41.

10. McBain H, Shipley M, Newman S. The impact of appearance concerns on depression and anxiety in rheumatoid arthritis. Musculoskeletal Care 2013;11:19-30.

11. Shen B, He Y, Chen H, Zhao C, Zhu L, Gao Y, et al. Body Image Disturbances Have Impact on the Sexual Problems in Chinese Systemic Lupus Erythematosus Patients. J Immunol Res 2015;2015:204513.

12. Boyington JE, Schoster B, Callahan LF. Comparisons of Body Image Perceptions of a Sample of Black and White Women with Rheumatoid Arthritis and Fibromyalgia in the US. Open Rheumatol J 2015;9:1-7.

13. Goodacre LJ, Candy FJ. 'If I didn't have RA I wouldn't give them house room': the relationship between RA, footwear and clothing choices. Rheumatology (Oxford) 2011;50:513-7.

14. Williams AE, Nester CJ, Ravey MI. Rheumatoid arthritis patients' experiences of wearing therapeutic footwear - a qualitative investigation. BMC Musculoskelet Disord 2007;8:104.

15. Skevington SM, Blackwell F, Britton NF. Self-esteem and perception of attractiveness: an investigation of early rheumatoid arthritis. $\mathrm{Br} \mathrm{J}$ Med Psychol 1987;60:45-52.

16. Cornwell CJ, Schmitt MH. Perceived health status, self-esteem and body image in women with rheumatoid arthritis or systemic lupus erythematosus. Res Nurs Health 1990;13:99-107.

17. Firth J, Nelson EA, Briggs M, Gorecki C. A qualitative study to explore the impact of foot ulceration on health-related quality of life in patients with rheumatoid arthritis. Int J Nurs Stud 2011;48:1401-8.

18. Radner H, Neogi T, Smolen JS, Aletaha D. Performance of the 2010 ACR/EULAR classification criteria for rheumatoid arthritis: a systematic literature review. Ann Rheum Dis 2014;73:114-23.

19. Taqui AM, Shaikh M, Gowani SA, Shahid F, Khan A, Tayyeb SM, et al. Body Dysmorphic Disorder: gender differences and prevalence in a Pakistani medical student population. BMC Psychiatry 2008;8:20.

20. Bao H, Yan P, Lonner B, Qiu Y, Ren Y, Zhu Z, et al. Validation of the Simplified Chinese Version of the Body Image Disturbance Questionnaire-Scoliosis. Spine (Phila Pa 1976) 2015;40:1155-60.

21. Sokka T, Kankainen A, Hannonen P. Scores for functional disability in patients with rheumatoid arthritis are correlated at higher levels with pain scores than with radiographic scores. Arthritis Rheum 2000;43:386-9. 
22. Kronisch C, McLernon DJ, Dale J, Paterson C, Ralston SH, Reid DM, et al. Brief Report: Predicting Functional Disability: One-Year Results From the Scottish Early Rheumatoid Arthritis Inception Cohort. Arthritis Rheumatol 2016;68:1596-602.

23. Koh ET, Seow A, Pong LY, Koh WH, Chan $\mathrm{L}$, Howe HS, et al. Cross cultural adaptation and validation of the Chinese Health Assessment Questionnaire for use in rheumatoid arthritis. J Rheumatol 1998;25:1705-8.

24. Prevoo ML, van Riel PL, van 't Hof MA, van Rijswijk $\mathrm{MH}$, van Leeuwen MA, Kuper $\mathrm{HH}$, et al. Validity and reliability of joint indices. A longitudinal study in patients with recent onset rheumatoid arthritis. $\mathrm{Br} \mathrm{J}$ Rheumatol 1993;32:589-94.

25. Emery P, Kavanaugh A, Bao Y, Ganguli A, Mulani $P$. Comprehensive disease control (CDC): what does achieving CDC mean for patients with rheumatoid arthritis? Ann Rheum Dis 2015;74:2165-74.

26. Li L, Wang HM, Shen Y. Chinese SF-36 Health Survey: translation, cultural adaptation, validation, and normalisation. J Epidemiol Community Health 2003;57:259-63.

27. Cordingley L, Prajapati R, Plant D, Maskell D,
Morgan C, Ali FR, et al. Impact of psychological factors on subjective disease activity assessments in patients with severe rheumatoid arthritis. Arthritis Care Res (Hoboken) 2014;66:861-8.

28. Wang W, Chair SY, Thompson DR, Twinn SF. A psychometric evaluation of the Chinese version of the Hospital Anxiety and Depression Scale in patients with coronary heart disease. J Clin Nurs 2009;18:2436-43.

29. Piyavhatkul N, Aroonpongpaisal S, Patjanasoontorn N, Rongbutsri S, Maneeganondh S, Pimpanit W. Validity and reliability of the Rosenberg SelfEsteem Scale-Thai version as compared to the Self-Esteem Visual Analog Scale. J Med Assoc Thai 2011;94:857-62.

30. Macsween A, Brydson G, Fox KR. Physical self perceptions of women with rheumatoid arthritis. Arthritis Rheum 2004;51:958-63.

31. Jorge RT, Brumini C, Jones A, Natour J. Body image in patients with rheumatoid arthritis. Mod Rheumatol 2010;20:491-5.

32. Kurt E, Özdilli K, Yorulmaz H. Body Image and SelfEsteem in Patients with Rheumatoid Arthritis. Noro Psikiyatr Ars 2013;50:202-8. 